Article

\title{
New way of synthesis of few-layer graphene nanosheets by the selfpropagating high-temperature synthesis method from bi- opolymers for large-scale production - a method of utilization waste from the woodworking industry for clean earth tomor-
} row

\author{
Alexander Voznyakovskii ${ }^{1}$, Aleksey Vozniakovskii ${ }^{2}$ and Sergey Kidalov 2,* \\ 1 Institute for Synthetic Rubber, Saint-Petersburg, Russia; voznap@mail.ru \\ 2 Ioffe Institute, Saint-Petersburg, Russia, alexey_inform@mail.ru \\ * Correspondence: Kidalov@mail.ioffe.ru
}

\begin{abstract}
For the first time, few-layer graphene (FLG) nanosheets were synthesized by the method of self-propagating high-temperature synthesis (SHS) from biopolymers (starch and lignin). We suggested that biopolymers (lignin, tree bark) and polysaccharides, in particular starch, could be an acceptable source of native cycles for the SHS process. The carbonization of biopolymers under the conditions of the SHS process was chosen as the basic method of synthesis. Chemical reactions, under the conditions of the SHS process, proceed according to a specific mechanism of nonsothermal branched-chain processes, which are characterized by the joint action of two fundamentally different process-accelerating factors - avalanche reproduction of active intermediate particles and self-heating. The method of obtaining FLG nanosheets included the thermal destruction of hydrocarbons in a mixture with an oxidizing agent. We used biopolymers as hydrocarbons and ammonium nitrate as an oxidizing agent. Thermal destruction was carried out in the mode of SHS, heating the mixture in a vessel at a speed of $20-30^{\circ} \mathrm{C} / \mathrm{min}$ to $150-200^{\circ} \mathrm{C}$ and keeping at this temperature for 15-20 min with the discharge of excess gases into atmosphere. A combination of spectrometric research methods, supplemented by electron microscopy data, has shown that the particles of the carbonated product powder in their morphometric and physical parameters correspond to FLG nanosheets. An X-ray diffraction analysis of the indicated FLG nanosheets was carried out, which showed the absence of formations with a graphite crystal structure in the final material. The surface morphology was also studied and the features of the IR absorption of FLG nanosheets were analyzed. It is shown that the developed SHS method makes it possible to obtain FLG nanosheets with linear dimensions of tens of microns and a thickness of not more than 1-5 graphene layers (several graphene layers).
\end{abstract}

Keywords: graphene; self-propagating high-temperature synthesis; few-layer graphene; biopolymers; starch; lignin; tree bark; carbonization of biopolymers; SHS; FLG

\section{Introduction}

Graphene materials such as graphene nanoplates (referred to in literature as graphene nanoplatelets or $\mathrm{GnP})$, graphene nanosheets $(\mathrm{GnS})$, graphene oxide $(\mathrm{GO})$, reduced graphene oxide (rGO), etc., are successfully used by researchers around the world in various promising areas. There are follows areas: as an additive in polymer [1] and metal composites [2] as an effective sorbent of radionuclides [3], as a material for creating supercapacitors [4], etc. However, despite the obtaining of interesting results using graphene materials, industrial implementation has not yet occurred due to the imperfection of the methods of their synthesis. 
Some methods, such as mechanical exfoliation of graphite using adhesive tape [5], chemical vapor deposition (CVD) on metal surfaces [6], epitaxial growth on single crystal $\mathrm{SiC}$ [7], chemical bonding reactions or exfoliation of graphite powder by oxidation in solution [8], sonication/intercalation [9], etc. currently in use, but mass production of graphene nanosheets is still a problem.

This is due to the lack of a simple, economical and at the same time environmentally friendly method for the production of high-quality graphene. A promising method for obtaining graphene structures, in addition to the above characteristics, should ensure their production in volumes sufficient for real use in specific engineering solutions. Currently, the most common is a three-stage process for obtaining graphene structures, including the oxidation of graphite to graphite oxide, subsequent separation to graphene oxide (GO), and, finally, chemical or thermal reduction to graphene.

The classical methods for obtaining graphene oxide are the Brodie method (proposed in 1859 by Benjamin Brodie) [10] and the Hammers method [11], which have been significantly improved by now. These methods are based on the exfoliation of graphite with concentrated acids followed by oxidation. As a result, a three-dimensional structure of graphite oxide is formed, which is then exfoliated to form graphene oxide nanosheets [12]. In the process of oxidation, in addition to the formation of oxygen-containing groups, defects in graphene oxide layers are also formed, which are defects in the carbon sublattice with the absence of two or more carbon atoms [13].

Reduced graphene oxide (rGO) can be obtained by both physical and chemical reduction of graphene oxide [14].

However, the need for one more technological operation significantly complicates the scaling process for obtaining graphene structures. It should also be noted that this variant of obtaining few-layer graphene leads both to a deterioration in the environmental parameters of the technique due to the use of toxic reducing agents and to a deterioration in its economic parameters. In addition, the impact of excess thermal energy and hazardous chemicals on the initial structure of graphite leads to significant structural disorder in the aromatic structure of the resulting graphene sheets (the presence of vacancy defects, the appearance of oxidized carbon atoms in the planar plane).

Another method is to obtain partially reduced GO, when environmentally friendly weak agents such as ammonia, galactose and $\mathrm{Na}_{2} \mathrm{CO}_{3}$ are used for the chemical reduction of GO. Thus, the main disadvantages of widely used approaches are:

- firstly, the use of hazardous chemicals for both humans and the environment;

- $\quad$ secondly, multistage and labor intensity;

- $\quad$ third, the need to supply a large amount of heat, and

- fourth, the presence of numerous defects in the microstructure of the obtained powder graphene nanosheets, which, in turn, lead to a noticeable deterioration of the unique properties of graphene.

Another approach to chemical exfoliation of graphite involves the intercalation of graphite with alkali metals such as potassium and lithium, followed by the exfoliation of such intercalated graphite compounds in several layers of graphene nanosheets $[15,16]$.

This approach is much simpler and more economical than the previous one. However, this is time consuming and hazardous strong acids are used throughout the entire process. This negatively affects the quality, productivity and economy of the produced powdered graphene nanosheets.

The complexity and environmental insecurity of the considered techniques makes them unpromising for obtaining graphene structures in volumes exceeding that one's required for interlaboratory studies. As a result, many research groups began to search for alternative methods for the synthesis of graphene structures.

For example to obtain graphene structures, a number of authors used a well-developed method of hydrothermal synthesis.

For example, in [17] reported the hydrothermal synthesis of GO particles using glucose as precursor. The hydrothermal method for the synthesis of nanodispersed particles is a fairly simple hardware, safe, controllable, environmentally friendly and inexpensive 
method. Accordingly, the complex of these parameters can provide great potential in the mass production of high quality GO.

In a similar work [18] reduced graphene oxide $(\mathrm{rGO})$ was obtained by hydrothermal synthesis using glucose as a precursor. The resulting rGO was further treated with ammonia, which made it possible to obtain particles of few-layer graphene nanosheets (FLG) in gram within an acceptable time interval.

In [19] reports on the possibility of bulk production of graphene nanosheets by pyrolysis of solid plastic waste. As a result of two-stage pyrolysis of plastic (at $400{ }^{\circ} \mathrm{C}$ in the presence of nanoclay, and then at $750{ }^{\circ} \mathrm{C}$ in a nitrogen atmosphere), a black powder was obtained. The study of the structure of the particles of the obtained powder by a complex of complementary spectral methods, supplemented by the method of microscopy, allowed the authors to compare their structures to graphene nanosheets.

In a number of works, the method of hydrothermal synthesis was supplemented by preliminary mechanical activation of the precursor by processing in a ball mill [20]. The authors report that the exfoliation efficiency can reach $92 \%$ (for particles $\leq 10$ graphene layers) without purification, and the total yield can reach $85.26 \%$.

In some cases, FLG nanosheets may be obtained directly by mechanical activation [21]. In this work, the authors applied the well-known elementary method of sonication of peanut shell (PS) to obtain few-layer nanosheets like FLG nanosheets. The authors report that the nanosheets obtained in this work have an extremely high specific surface area (up to $2070 \mathrm{~m}^{2} / \mathrm{g}$ ) and developed microporosity (up to $1.33 \mathrm{~cm}^{3} / \mathrm{g}$ ).

From our best knowledge we can conclude that, in general, to obtain graphene structures from precursors of various natures, including both natural sources (fossil graphite, natural biomass) and precursors of technogenic origin (waste from the polymer chemistry industry, waste from the processing industry) use a combination of processes (sequentially or simultaneously) of thermal destruction and oxidation processes of precursors. In addition, a common feature of the considered techniques is the mechanism by which FLG particles are formed - the "top-down" mechanism, that is, in one form or another, it uses the exfoliation processes of the initial precursor to single graphene structures.

The disadvantages of the described "top-down" techniques can be attributed to both their insufficient productivity and the low quality of synthesized graphene structures due to the presence of defects in their honeycomb structure.

These shortcomings of top-down techniques have prompted many research groups to switch to «bottom-up» techniques for producing 2D carbon nanostructures. The most commonly cited bottom-up methodology in the literature is the CVD method [22]. However, the «bottom-up» approach, which makes it possible to obtain individual graphene sheets of high quality (low-defect), also does not allow providing the required productivity of the synthesis of graphene structures.

In this paper, we want to report on a new technique for the synthesis of large volumes of FLG nanosheets based on the self-propagating high-temperature synthesis (SHS) method. Physically, SHS is a process of moving a wave of a strong exothermic reaction through a mixture of reagents (oxidizing agent and reducing agent), in which heat release is localized in the layer and is transferred from layer to layer by means of heat transfer. Typical characteristics of the SHS process are following: flame front propagation speed $(0.1-20) \mathrm{cm} / \mathrm{s}$; maximum combustion temperature - (2300-3800) $\mathrm{K}$; the heating rate of matter in the wave is $\left(10^{3}-10^{6}\right) \mathrm{deg} / \mathrm{s}$. [23].

The SHS method, from our point of view, to a certain extent can be considered an analogue of detonation synthesis used to obtain 3D carbon nanostructures - detonation nanodiamonds. Detonation synthesis implies the occurrence of two sequential processes organized in a certain way: explosive decomposition of, used as a precursor, a mixture of two cyclic organic compounds (trinitrotoluene and cyclotrimethylenetrinitramine) and the subsequent process of self-organization of degradation products in the front of shock wave. Under specific conditions of detonation synthesis, under the influence of extremely high pressure and temperature, 3D carbon nanostructures - detonation nanodiamonds are formed. 
The implementation of detonation synthesis shows that there are no theoretical restrictions on the synthesis of 2D carbon nanostructures in the sequence of processes "destruction - self-organization". Chemical reactions under the conditions of the SHS process proceed according to a specific mechanism of nonisothermal-branched chain processes, which are characterized by the combined action of two fundamentally different factors accelerating the process: avalanche multiplication of active intermediate particles and selfheating. Branched chain ignition, in contrast to thermal, is caused by avalanche multiplication of active intermediate products - free atoms, radicals and sometimes-excited particles - in their rapid reactions with the initial reagents and among themselves [24].

When choosing a carbonization technique, we proceeded from the fact that the advantage of using the SHS process over the pyrolysis and hydrothermal carbonization processes traditionally used for carbonization of biopolymers [18] is the simplicity of the hardware design of the method. In addition, there are high synthesis rates, the ability to carry out synthesis without a constant supply of energy from external power sources, the ability to carry out synthesis in any atmosphere or in a vacuum, the absence of fundamental large-scale limitations $[25,26]$.

Based on the analogy with detonation synthesis, we assumed that biopolymers with a cyclic macrochain structure could be an acceptable source of native cycles (precursors) for the SHS process.

In formulating this assumption, we relied on the comparison of graphene to a specific macromolecule, the native element of which (monomer) can be a cycle of six carbon atoms - hexagon. An acceptable source of hexagons can be plant polymers, the macromolecules of which are either formed by cyclic structures (starch, cellulose), or whose macromolecules are formed by aromatic sequences (lignin). The choice of the source of such cycles, as well as the development of a technique for initiating their "bottom-up" self-organization processes, would make it possible to obtain 2D carbon nanostructures on a scale required for real application.

Let us clarify that biopolymers of plant origin include polymers generated by plants in the course of their metabolism. The most well-known plant biopolymers that can be obtained in an individual state include starch, lignin, cellulose. However, these biopolymers are part of complex plant biocomplexes, from which they can be isolated as a result of certain technological operations.

Moreover, if starch and cellulose are the target product, then lignin is obtained because of the processing of woody biomass during the technological process of obtaining cellulose. In this case, lignin, which is a complex, irregularly constructed, resistant to decomposition, insoluble in water and organic solvents, a high molecular weight polymer with branched macromolecules of a cyclic structure, is a difficultly utilized plant polymer. As a result, to date, a critical volume of its storage has been accumulated, the uncontrolled further growth of which can lead to an irreversible negative impact on the environment. Accordingly, the utilization of plant biopolymers, which are not a target resource, is one of the most important tasks of environmental management. In this case, they are considered as secondary raw materials [27].

Thus, the choice of natural biopolymers as a precursor for obtaining graphene nanostructures will lead not only to the possible production of a valuable product - graphene structures, but also, to a certain extent, will contribute to the elimination of environmental problems caused by such wastes.

\section{Preparation and carrying out the process of the SHS process}

Lignin carbonization was carried out under the conditions of the process of SHS method. The process was carried out using a laboratory reactor, which is a quartz vessel ( $1 \mathrm{~L}$ capacity) with a heating element in the lower part, which provides the initial heating of the reaction zone to the temperature required to initiate the process $\left(220^{\circ} \mathrm{C}\right)$. Temperature control in the reaction zone was carried out using a thermocouple. The preparation 
of the synthesis included a number of sequential stages. The pre-dried lignin was transferred to a ball mill and processed for 15 minutes. Next, a mechanical mixture of an oxidizing agent (ammonium nitrate) and a precursor (lignin) was prepared in a weight ratio of 1:1. The prepared mechanical mixture of the oxidizing agent and the precursor was placed in a "drunken barrel" mixer and processed for 15 minutes. Then the resulting crushed mixture was transferred into a reactor preheated and purged with a dry argon flow. The beginning and end of the reaction was judged by the beginning and end of the evolution of gaseous reaction products. The duration of the process is $5-8$ minutes. The yield of the carbonization reaction (according to the precursor) is 30-35\% wt. The rest of the reaction mass passes into the gas phase and is captured in a trap cooled with liquid nitrogen. The process flow diagram is shown in Figure 1.

The methodology for producing FLG nanosheets from wood waste includes several steps, as shown in Figure 1.

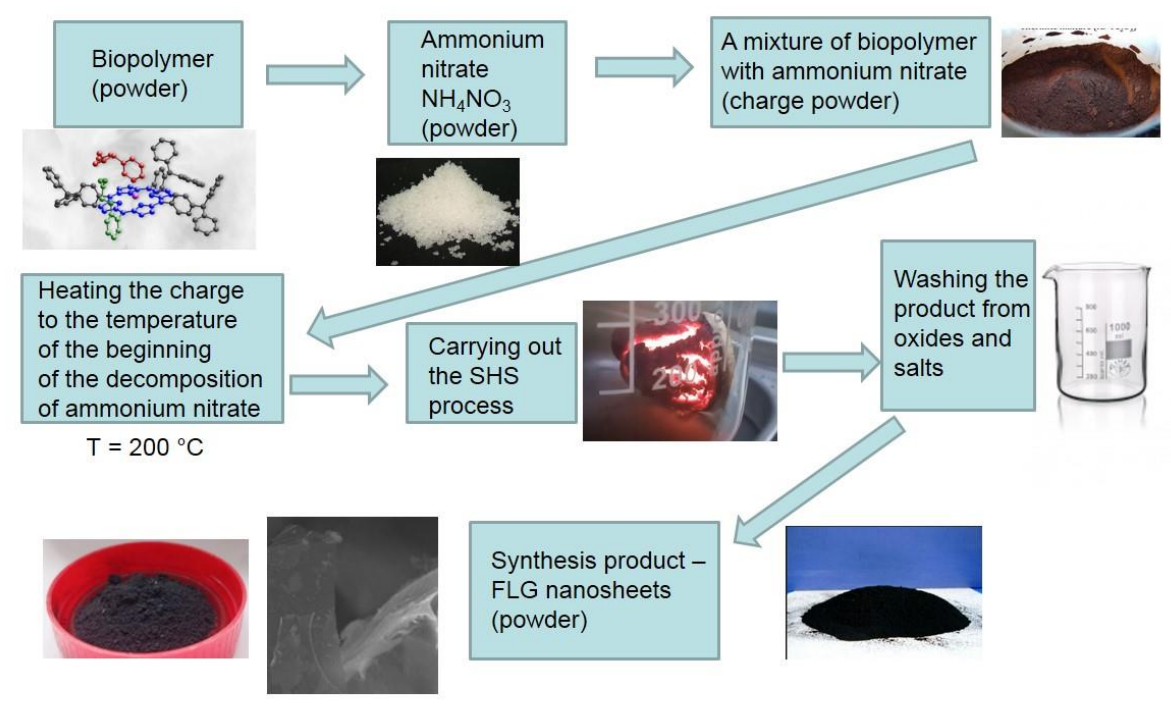

Figure 1. Process flowchart.

All raw materials were crushed (grain crusher "Kolos 2M") and dried to constant weight in a ShS-40-02 drying oven at $80^{\circ} \mathrm{C}$. The dried powder was finally grind in a laboratory planetary mill LP-1- HT Machinery, (Japan) and sift through a sieve to a particle size of the order of 100 microns ( \pm 10 microns).

After completing these elementary cleaning and drying steps, the waste and ammonium nitrate (average particle diameter $10 \mu \mathrm{m}$ ) were thoroughly mixed using a "drunken barrel" mixer in a certain ratio.

The mixture of the initial components prepared in this way was slowly heated to a temperature of about $150-200{ }^{\circ} \mathrm{C}$ in an oil bath in an air atmosphere before the start of the decomposition of ammonium nitrate.

Schematic representation of the setup for SHS and the course of the SHS process shown on Figure 2. 


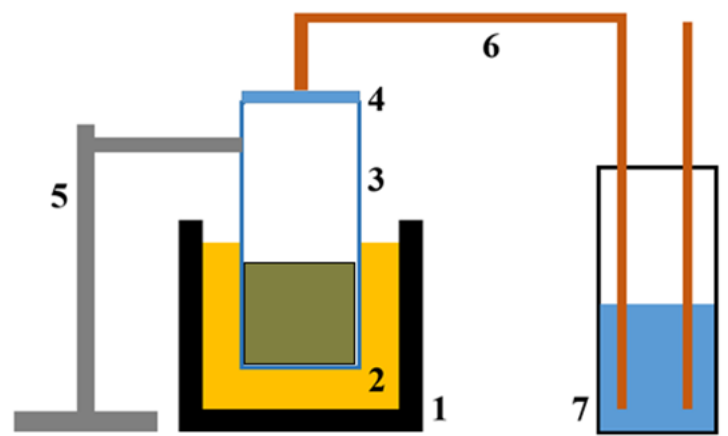

(a)

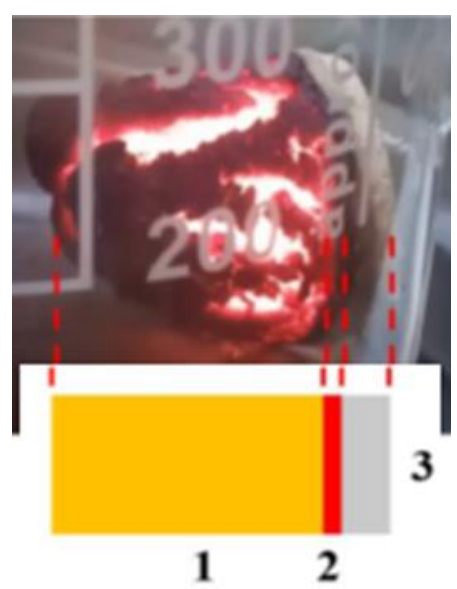

(b)

Figure 2. Schematic representation of the setup for SHS (a) and the course of the SHS process (b). (a) - 1 - oil bath, 2 - PVA300 oil, 3 - reactor with charge, 4 - reactor cover, 5 - tripod-holder, 6 - gas exhaust system, 7 - water filter. (b) - The front of the wave of the SHS process is visible, 1 - reacted mixture (product), 2 - reaction zone (SHS wave front), 3 - unreacted mixture (initial charge).

The main feature of carbonization in the process of rapid pyrolysis (a process usually used for the carbonization of plant polymers) is that rapid pyrolysis is carried out during the thermal de-composition of woody biomass in the absence of an oxidizing agent.

The fundamental difference between the carbonization of plant polymers (mainly woody biomass and products of its processing) under the conditions of the SHS process that in our case, there are active oxidants - nitrogen and oxygen obtained during the decomposition of ammonium nitrate. This is the most important difference.

Another significant difference between the processes of fast pyrolysis and the SHS process we use is that the carbonization process under conditions of fast pyrolysis is carried out with a constant supply of thermal energy, that is, the process is self-accelerating [28], while the SHS process is self-inhibiting [23].

Based on these two differences in the considered carbonization processes, it was assumed that, under the conditions of the SHS process, the carbonization of plant biomass would proceed only to a solid carbonized product. This moment of the SHS process we use creates the basis for the nucleation of FLG nanosheets with the secession of all gas oxides and metal oxides.

After the completion of the SHS process, a black powder was obtained. The black powder collected in this way at the end of the SHS process was washed with distilled water to remove water-soluble products (oxides, metal nitrates, etc.). The resulting FLG nanosheets were either dried or left in an aqueous suspension. Experimental conditions are optimized to synthesize FLG nanosheets with up to five layers.

\section{Characterization}

To confirm the successful synthesis of FLG nanosheets, various spectroscopic research methods have been performed, SEM and TEM images was obtained.

Raman spectroscopy (Confotec nr500, $532 \mathrm{~nm}$, SOL Instruments, Belarus) at an exciting laser length of $532 \mathrm{~nm}$ was used to demonstrate the vibrational properties of FLG nanosheets.

Fourier transform infrared spectroscopy was performed using an INFRALUM FT-08 instrument, Russia to study functional groups on the surface of FLG nanosheets. X-ray diffraction (XRD) was performed using a XRD-7000 ((CuK $\alpha$ - radiation, $\lambda=0,154051 \mathrm{~nm}$, Shimadzu, Jupan).

SEM and TEM studies of the morphology and structure of FLG nanosheets were carried out using the TESCAN Mira-3M (Czech) with Electron Dispersive X-ray Spectroscopy (EDX) device attachment (Oxford instruments X-max) and FEI Tecnai G2 30 S-TWIN (50 $\mathrm{kV}$ ) microscope. UV-VIS spectra investigated on Shimadzu-IS, Japan. The studies based 
on the method of the X-ray photoelectron spectroscopy were conducted on the instrument Thermo Scientific K-alpha (USA).

\section{Results}

To evaluate the success of the above experimental strategy for synthesizing FLG nanosheets using the SHS method, we used various methods to validate the synthesis of FLG nanosheets.

\subsection{SEM and TEM studies}

The material synthesized by the SHS method was a black, highly dispersed, highly volatile powder. The structure of synthesized FLG nanosheets obtained using TEM is shown in Figure 3.

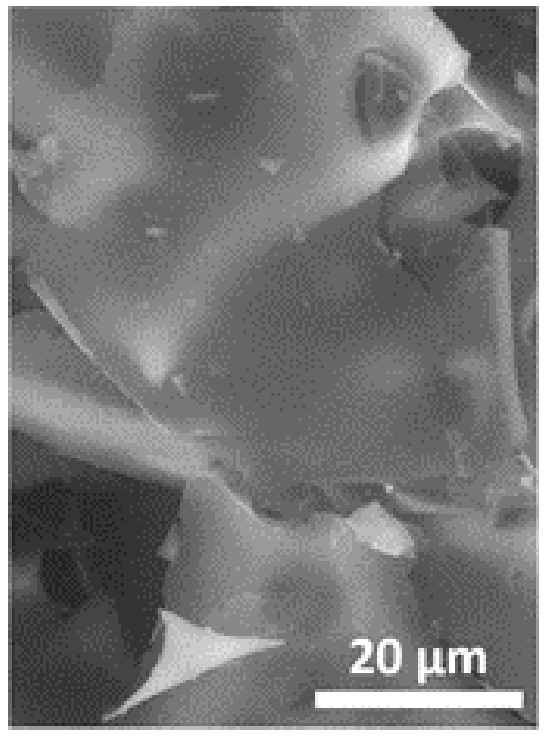

(a)

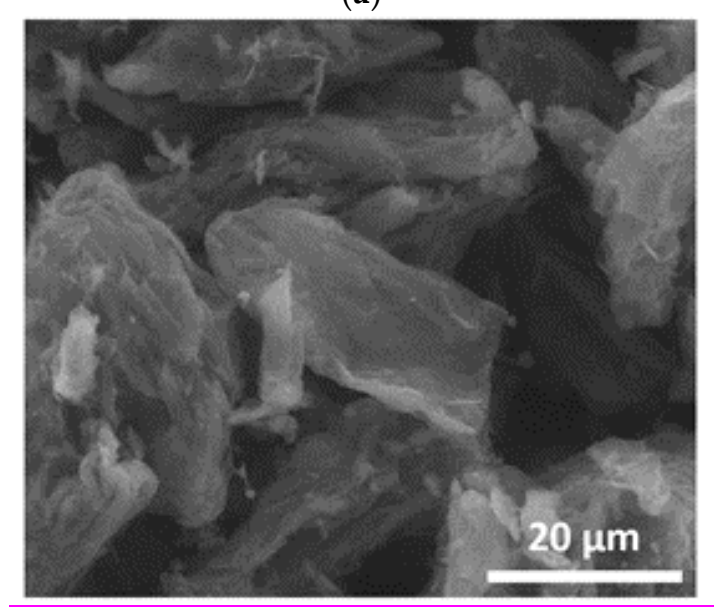

(c)

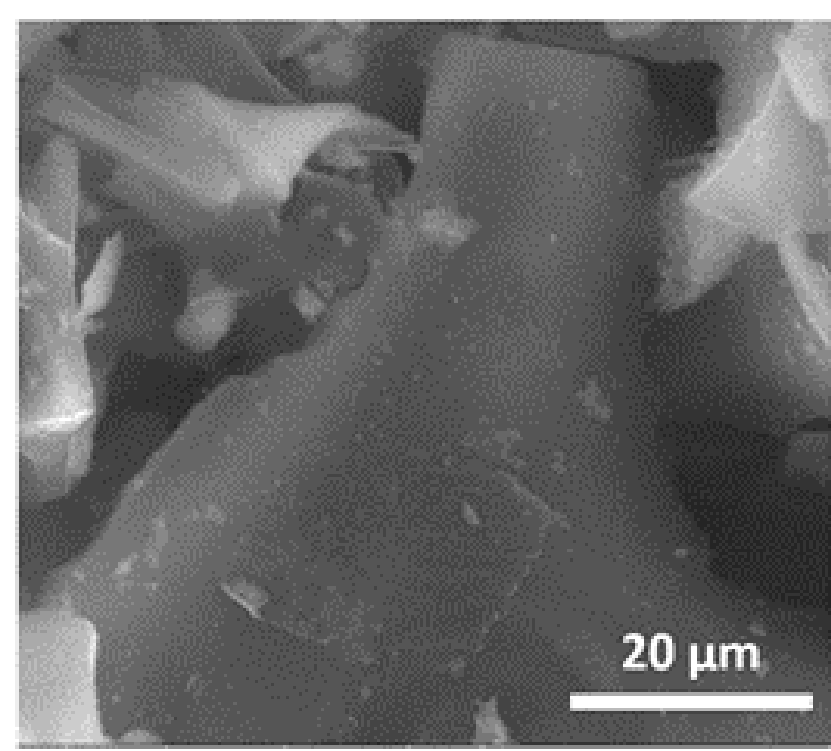

(b)

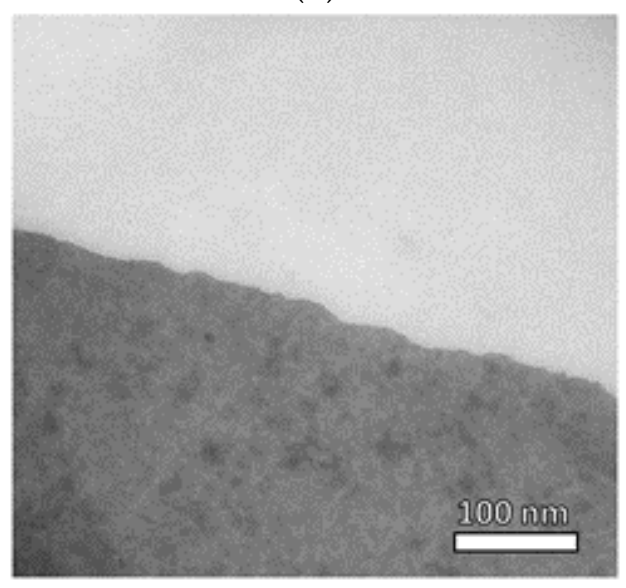

(d) 


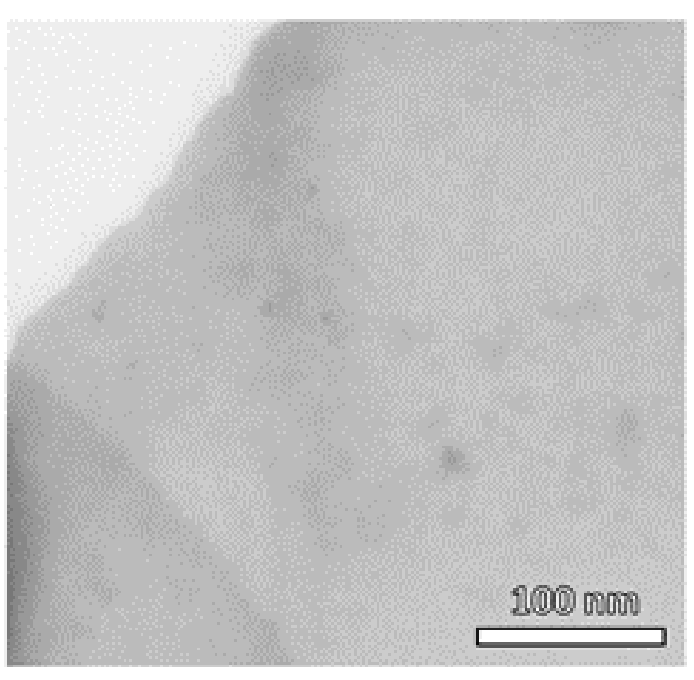

(e)

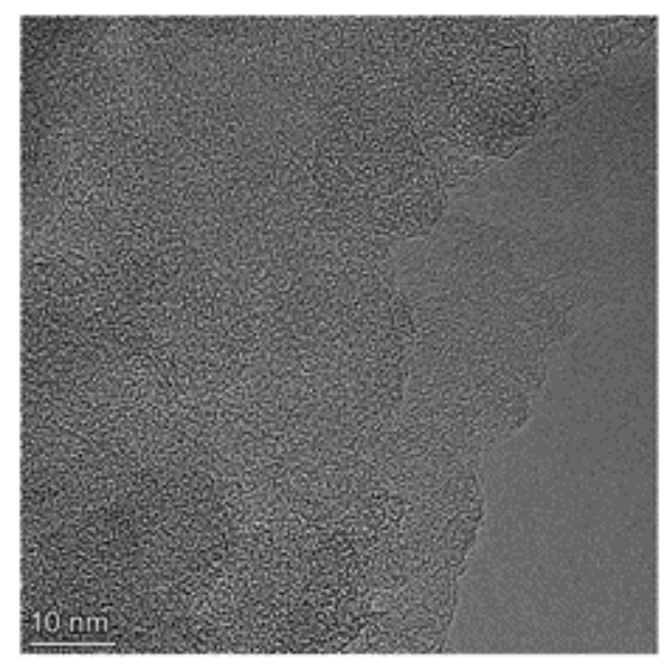

(f)

Figure 3. SEM and TEM images of synthesized FLG nanosheets. (a), (b) and (c) SEM images - sample synthesized from glucose, starch, and cellulose respectively. Linear scale: a-c - $20 \mu \mathrm{m}$; (d), (e) and (f) - TEM images - sample synthesized from glucose, starch, and cellulose respectively. Linear scale: $\mathrm{d}-100 \mathrm{~nm}$, e - $100 \mathrm{~nm}$ and f $-10 \mathrm{~nm}$.

As can be seen from Figure 3, the FLG nanosheets synthesized by the SHS method have linear dimensions of about 10-30 $\mu \mathrm{m}$. It should be noted that the obtained FLG nanosheets are not strictly plane-parallel and have curvatures. However, Figure $3 \mathrm{~b}$ shows a sheet (possibly single layer) of FLG nanosheets.

As can be seen from Fig. 3, the synthesized particles are thin, which indicates that the number of graphene layers does not exceed 5 .

\subsection{EDX research}

The EDX studies (Figure 4) showed the high purity of the FLG nanosheets obtained by the SHS method.

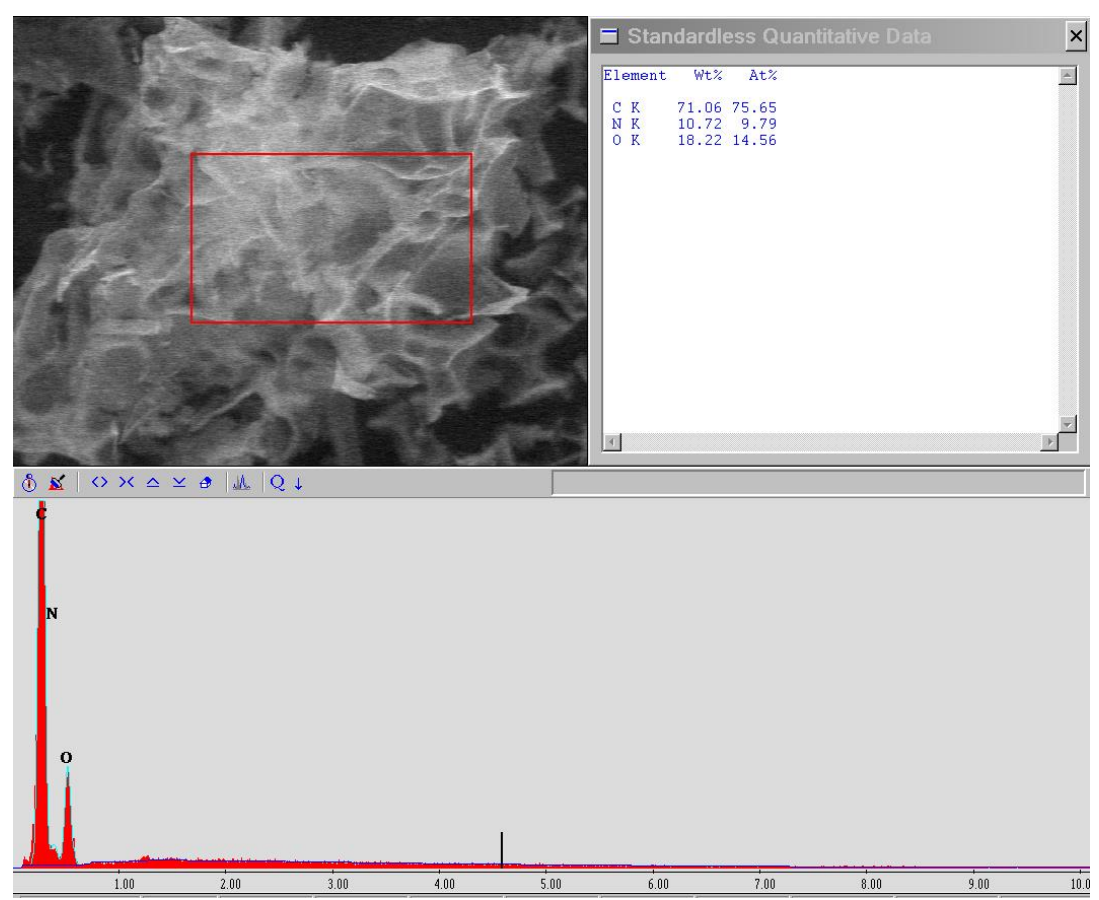

Figure 4. EDX study of synthesized FLG nanosheets from glucose.

From the above data on the study of a sample obtained from glucose, it can be concluded that there are no impurities of metals or other elements. The main impurities are 
oxygen and nitrogen (due to the presence of an oxidizing agent - ammonium nitrate in the SHS process) contained in an amount of no more than 10 and 15 At.\%, respectively. FLG nanosheets produced from other starting materials had the same impurity purity.

\subsection{Raman spectroscopy data}

Raman spectroscopy was performed to confirm the synthesis of exactly FLG nanosheets and to assess their quality (Figure 5).

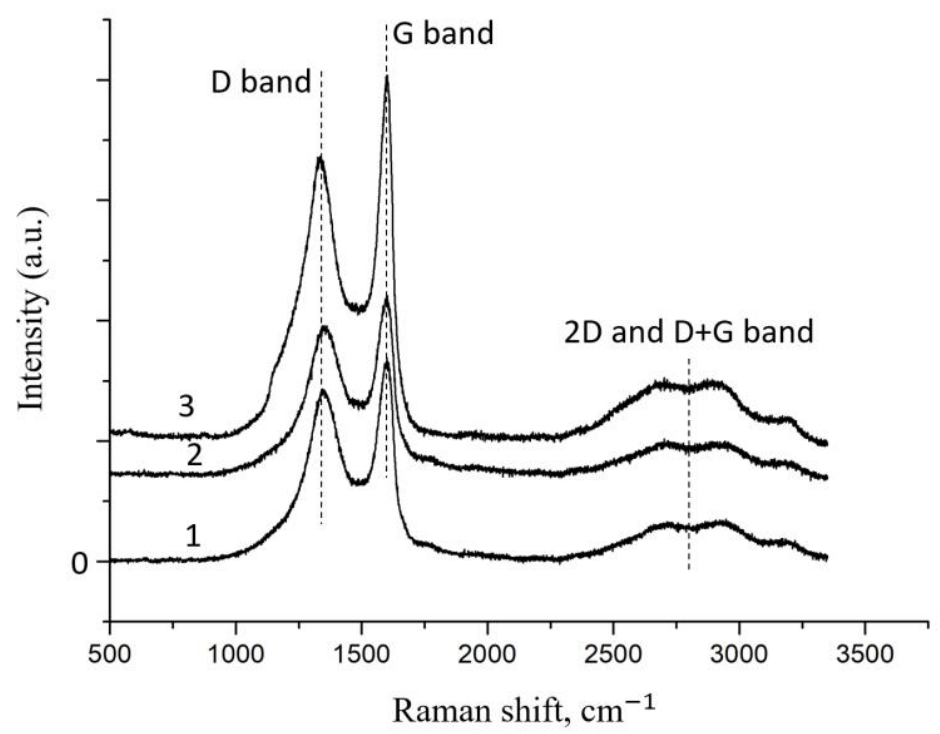

Figure 5. Raman spectra of FLG nanosheets obtained in the SHS process under various conditions and from various precursors. 1 - FLG nanosheets derived from glucose, 2 - FLG nanosheets derived from cellulose, c - FLG nanosheets derived from lignin.

Various active modes are observed in the synthesized samples. The Raman spectra of all samples contain four peaks characteristic of graphite materials, corresponding to $\mathrm{D}$ $\left(1310-1430 \mathrm{~cm}^{-1}\right), G\left(1570-1595 \mathrm{~cm}^{-1}\right), 2 \mathrm{D}\left(2500-2800 \mathrm{~cm}^{-1}\right)$ and D+G $\left(3000 \mathrm{~cm}^{-1}\right)$. The $G$ band arises from the planar vibrations of the aromatic carbon sp2. The $2 \mathrm{D}$ band is a second order Raman characteristic indicative of crystalline graphite materials and is sensitive to the aromatic $\mathrm{C}$-structure. The $\mathrm{D}$ and $\mathrm{D}+\mathrm{G}$ bands represent Raman features caused by lattice disorder. Disorder in the graphite lattice occurs due to defects associated with vacancies, grain boundaries, carbon particles sp3 and the inclusion of $\mathrm{O}$ in the aromatic sp2 Cnetwork [29].

It was found that comparison of the $2 \mathrm{D}$ and $\mathrm{D}+\mathrm{G}$ Raman bands can indirectly control the electronic structure ( $\pi$-orbitals). Band D shows partially disordered structures of the $\mathrm{sp}^{2}$ carbon atom associated with structural defects, and band $\mathrm{G}$ shows the degree of graphitization. The low intensity ratio of the $G$ band to the $\mathrm{D}$ band $\left(\mathrm{I}_{\mathrm{G}} / \mathrm{ID}_{\mathrm{D}}=1.10\right.$, graphene nanosheet) indicates the existence of randomly arranged graphene nanosheets. In addition, a wide $2 \mathrm{D}$ band also appears at $2790 \mathrm{~cm}^{-1}$, confirming the presence of few-layer graphene nanosheets [30].

The G-band is Raman-active for the $\mathrm{sp}^{2}$-hybridized carbon material, while the Dband is activated only if the defects are involved in double resonance Raman scattering of light near the $K$ point of the Brillouin zone. Thus, our results show that the average size of $\mathrm{sp}^{2}$ domains changes significantly in samples prepared from lignin relative to samples obtained from cellulose and glucose, since the intensity ratio ID/IG is usually used to estimate the size of the $\mathrm{sp}^{2}$ domain of graphite-based materials.

In addition to the $\mathrm{G}$ and $\mathrm{D}$ bands, there are two weaker Raman bands, called $2 \mathrm{D}$ and $\mathrm{D}+\mathrm{G}$, located in the $2700-3000 \mathrm{~cm}^{-1}$ region. The $2 \mathrm{D}$ band is Raman active for crystalline graphite materials and is sensitive to the-band in the electronic structure of graphite, while the $D+G$ combination mode is caused by disorder. The $\mathrm{I}_{2 \mathrm{D}} / \mathrm{I}_{\mathrm{D}+\mathrm{G}}$ intensity ratios show that the recovery of graphite electronic interfacing for glucose-derived samples is significant. 
Thus, it can be concluded that the FLG nanosheets obtained by the SHS method have structural defects and have a slightly curved surface with kinks, breaks and bends.

\subsection{X-ray structural analysis}

To prove the absence of graphite nanostructures in the obtained material (graphite, thermally expanded graphite, nanographite, etc.), an X-ray diffraction analysis was carried out, the results of which are shown in Figure 6.

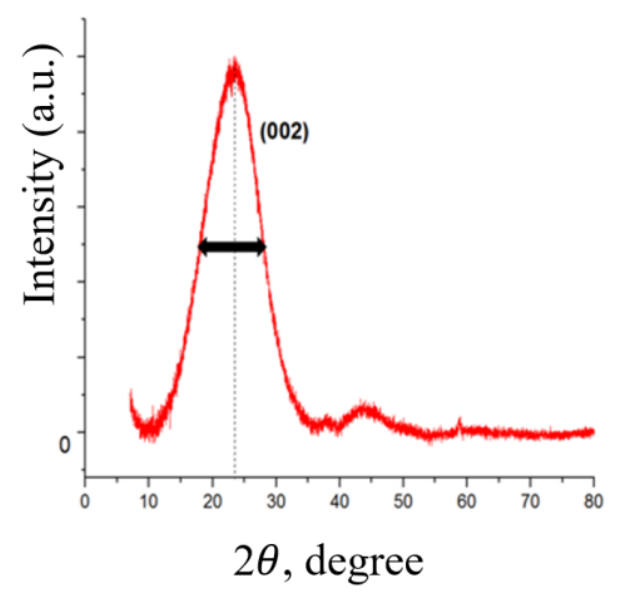

Figure 6. Results of X-ray spectroscopy studies of the synthesized FLG of graphene nanostructures. Typical diffractogram.

A halo in the range from 10 to 35 degrees and reflections at $\sim 43$ degrees strictly proves the absence of any graphite materials, the diffraction patterns of which are characterized primarily by a narrow and intense peak in the region of 26 degrees, as well as a narrow peak with low intensity in the region of 54- 55 degrees [31]. As can be judged from the data in Figure 6, the form of the obtained diffraction pattern fully corresponds to the typical curves obtained on 2D carbon structures - graphene nanoplatelets [32].

As seen from Figure 6, a typical diffraction pattern has a wide amorphous halo typical of graphene nanostructures. The absence of any narrow peaks in the diffraction patterns also indicates the absence in our FLG nanosheets of any water-insoluble impurities that could form during SHS process, which is consistent with the EDX data (Figure 4). We also estimated the number of layers in the synthesized FLG nanosheets using the formula $\mathrm{N}=(\mathrm{L} / \mathrm{d})$, where $\mathrm{N}$ is the number of layers; $\mathrm{L}$ is the thickness of the stack of graphene structures; $d$ - interlayer distance [33]. The thickness of the stack of graphene structures was calculated using the Scherrer formula [34].

$$
L=n \lambda / \beta \cdot \operatorname{Cos}(\theta)
$$

where $L$ is the thickness of the pack; $\lambda=0.154051 \mathrm{~nm}$ - radiation wavelength; $\theta$ - scattering angle; $\beta$ - physical line broadening in the diffractogram in radians (on a $2 \theta$ scale); $\mathrm{n}$ - particle shape factor equal to 1.

The number of layers in FLG nanosheets obtained by the SHS method from biopolymers is set as a value not exceeding five layers. 


\subsection{FTIR research data}

To assess the functional composition of the edges of FLG nanosheets, FTIR studies were carried out (Figure 6). It is show that in addition to the typical GNP groups $\mathrm{CH}, \mathrm{C}$ $\mathrm{OH}, \mathrm{CO}-\mathrm{C}$ (edge groups), and $\mathrm{C}=\mathrm{C}$ (the bond between carbon atoms ), the sample contains $\mathrm{C} \equiv \mathrm{N}$ groups, the source of nitrogen in which is the oxidizing agent used in the synthesis.

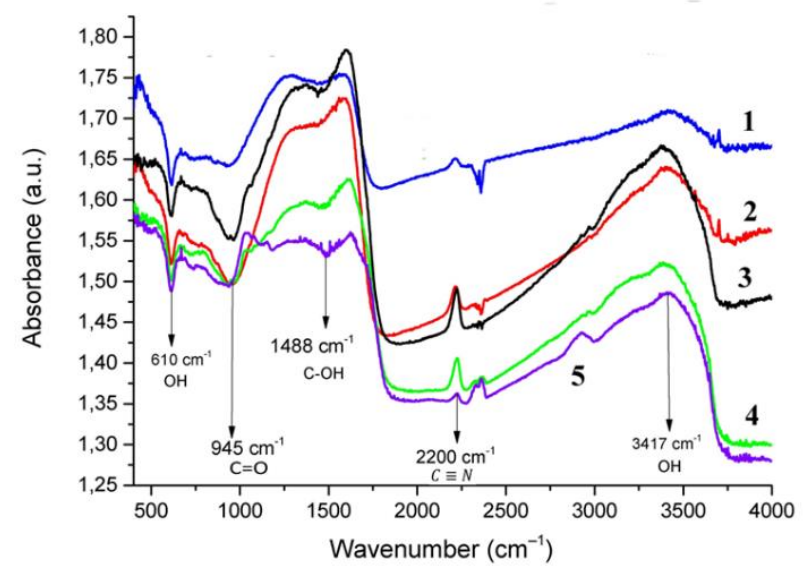

Figure 7. Results of the study of synthesized FLG nanosheets by FTIR spectrometry. The initial charge as a biopolymer includes 1 - cellulose, 2 - glucose, 3-5 - starch. Spectra 3-5 were obtained on FLG nanosheets obtained in a starch-based process under various conditions of the SHS process.

It can be seen that, depending on the initial biopolymer, the content of $\mathrm{C} \equiv \mathrm{N}, \mathrm{C}-\mathrm{O}$ and $\mathrm{C}-\mathrm{OH}$ groups on the surface of FLG nanosheets changes. Similar results were obtained using lignin as starting material.

\subsection{Study of the Specific surface area.}

In addition, in order to characterize the synthesized FLG nanosheets, studies were carried out by the method of gas (helium) pycnometry, as well as the measurement of the specific surface by the BET method, the results of which are presented in Table 1.

Table 1. Specific surface area and true density of FLG nanosheets synthesized from various biopolymers.

\begin{tabular}{ccc}
\hline Sample & $\begin{array}{c}\text { Specific surface } \\
\mathbf{m}^{2} / \mathbf{g}\end{array}$ & True density, $\mathbf{g} / \mathbf{c m}^{\mathbf{3}}$ \\
\hline From cellulose & 672 & 2,13 \\
From glucose & 512 & 2,11 \\
From Lignin & 500 & 2,12 \\
\hline
\end{tabular}

As can be seen from the data presented in Table 1, all samples of FLG nanosheets have a developed specific surface area $\left(>500 \mathrm{~m}^{2} / \mathrm{g}\right)$. Although GNP samples can have a specific surface area of up to $750 \mathrm{~m}^{2} / \mathrm{g}$ [35], usually such high values of specific surface are typical for GNP, with a particle size less than 2 microns [36]. However, in our case, such a developed specific surface area was obtained for FLG nanosheets with linear dimensions of tens of microns (Figure 3). It should be noted that, in contrast to the specific surface area, the true density of the samples does not depend on the type of the initial biopolymer.

Thus, based on the set of data obtained, we can confidently conclude that the structure of the particles obtained in the work corresponds to the structure of FLG nanosheets.

\subsection{UV-VIS research data}

Studies of optical absorption in the UV-VIS range have shown that the spectrum corresponds to graphene structures (Figure 8). The absorption peak lies in the $260 \mathrm{~nm}$ range, which indicates the practical absence of absence of oxygen in on the plane of FLG 
nanosheets. It can be noted that for glucose, the peak has a maximum at $264 \mathrm{~nm}$, for cellulose at $266 \mathrm{~nm}$, and for starch, it is shifted to $267 \mathrm{~nm}$.

It could be attributed to $\pi-\pi^{*}$ transitions of aromatic $C-C$ bonds, which is in agreement with report for high-quality graphene dispersions by liquid-phase exfoliation and corresponds to FLG nanosheets with up to 5 layers [37].

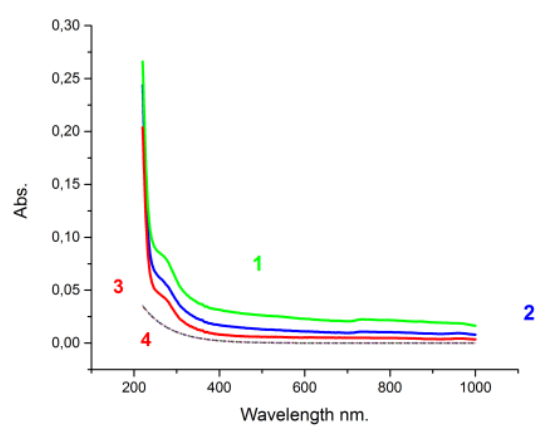

(a)

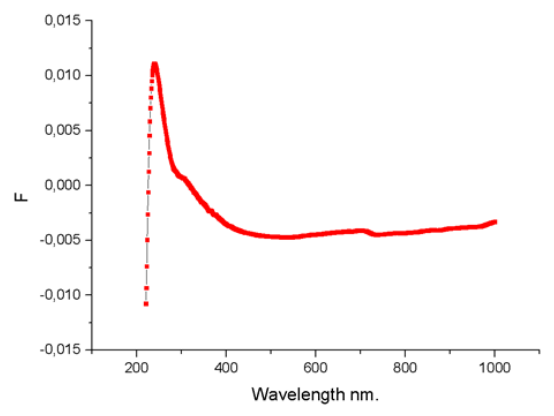

(b)

Figure 8. UV-VIS spectra of FLG nanosheets. (a) - UV-VIS absorption spectra in FLG nanosheets. 1 - glucose, 2- starch, 3cellulose, 4 - background spectrum. (b) - graph for FLG nanosheets from starch with the background subtracted on residual hydrocarbons that may be present in graphene nanosheets.

\subsection{XPS spectra}

Figure 9 shows that there are peaks typical for graphene nanostructures: $\mathrm{C}=\mathrm{C}\left(\mathrm{sp}^{2}\right.$, 284.4) C-OH (285.4), OCO/C-OH (286.5), C=O (287.4), OC=O (289), and also the C-N (285.9) peak is observed in the spectrum [38]. The appearance of the C-N peak is due to using a nitrogen-containing oxidant in the synthesis of FLG nanosheets by the SHS method. 


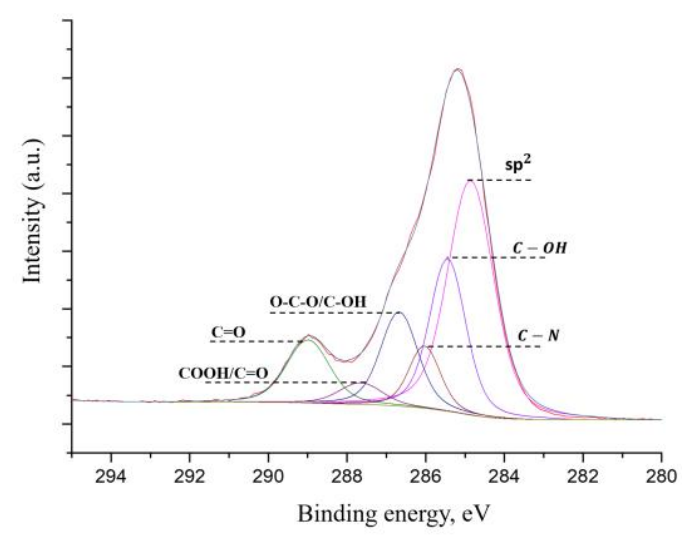

(a)

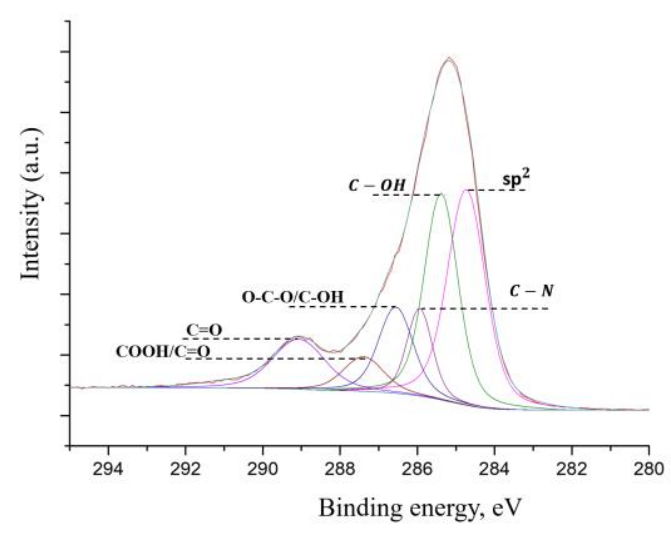

(b)

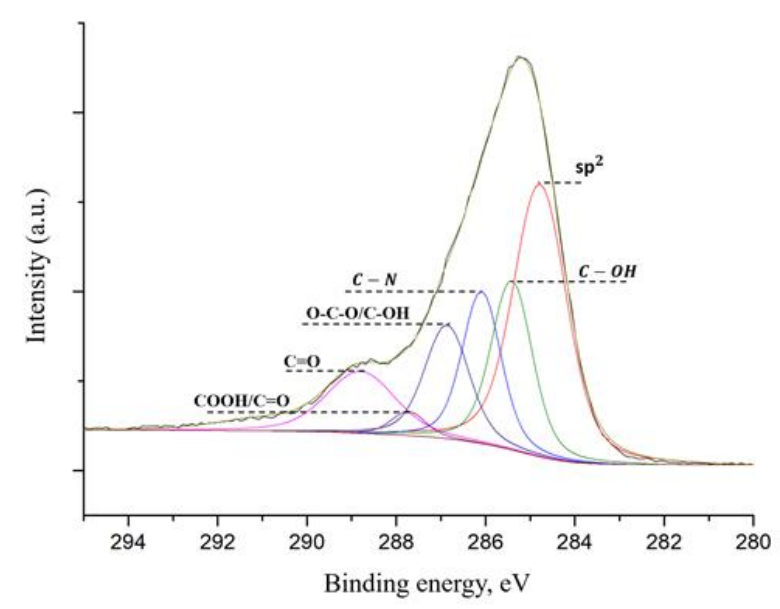

(c)

Figure 9. XPS spectra of FLG nanosheets. (a) - glucose,(b)- starch, (c)- cellulose.

\section{Discussion}

We report on an environmentally friendly, reliable and cost-effective method for mass-producing FLG nanosheets from wood waste.

Raman spectroscopy, TEM, FTIR, UV-VIS and EDX spectroscopy were performed to confirm the synthesis of FLG nanosheets, including identification of functional groups and quantitative analysis of elements. The proposed SHS method is very effective and allows converting waste from the wood processing industry into FLG nanosheets with a finished product yield of about $40 \%$.

Thus, the results of our investigation showed that the SHS method could be used for to obtain FLG nanosheets from polysaccharides (starch, lignin, etc.) as precursor. The FLG nanosheets obtained are characterized with linear dimensions of tens of microns and several (up to 5) graphene layers thick.

Conducted research allowed us to conclude that the imperfection of the lattice of FLG nanosheets, obtained by carbonization of biopolymers in the process of self-propagating high-temperature synthesis, are free from Stone-Wales defects but mainly determined by vacancy defects.

Method developed by us allows obtaining large volumes of FLG nanosheets. The use of the developed material in areas requiring large volumes of raw materials and not requiring high-quality single layer graphene, namely, when creating composite materials, as an effective sorbent and other areas will be possible.

Moreover, as our preliminary experiments demonstrate, graphene synthesized by SHS method can be used for various other applications, such as a material for supercapacitors, for fuel cells, as a filler for polymer and metal nanocomposites, nanofluids based on water and organic solvents for cooling systems, etc. 


\section{Conclusions}

Thus, a new high-performance VVK method has been developed for the synthesis of few-layer graphene nanostructures of a large area from biopolymers, including waste from the woodworking industry - lignin and tree bark. The VVK method will make it possible to obtain graphene nanostructures in a cheap way in large quantities while solving the environmental problem existing in the world. The VVK method is a breakthrough in the technology and production of graphene nanosheets.

Author Contributions: Conceptualization, A.V.; methodology, A.V. and S.K.; validation, A.V., A.V. and S.K.; investigation, A.V., A.V. and S.K.; resources, A.V., A.V. and S.K.; data curation, A.V. and S.K.; writing-original draft preparation, A.V., A.V. and S.K.; writing-review and editing, A.V., A.V. and S.K.; visualization, A.V., A.V. and S.K.; supervision, A.V., A.V. and S.K.; project administration, A.V. and S.K.; funding acquisition, A.V., and S.K. All authors have read and agreed to the published version of the manuscript.

Funding: This research was funded by Russian Foundation for Basic Research within the framework of scientific project No. 18-29-24129 MK.

Acknowledgments: The authors are grateful to Larisa Sharonova for carrying out the UV-VIS research and Alexander Vul' for constant attention to work, fruitfully discussions and comprehensive support.

Conflicts of Interest: The authors declare no conflict of interest. The funders had no role in the design of the study; in the collection, analyses, or interpretation of data; in the writing of the manuscript, or in the decision to publish the results.

\section{References}

1. Chatterjee S., Nafezarefi F., Tai NH, Schlagenhauf L., Nüesch FA, Chu BTT Size and synergy effects of nanofiller hybrids including graphene nanoplatelets and carbon nanotubes in mechanical properties of epoxy composites Carbon 2012, Vol. 50. N 15. P. 5380. doi 10.1016 / j.carbon.2012.07.021

2. Moghadam A.D., Omrani E., Menezes P.L., Rohatgi P.K. Mechanical and tribological properties of self-lubricating metal matrix nanocomposites reinforced by carbon nanotubes (CNTs) and graphene - A review Composites Part B: Engineering 2015. Vol. 77. P. 402. doi 10.1016 / j.compositesb.2015.03.014

3. Wang X., Chen Z., Wang X. Graphene oxides for simultaneous highly efficient removal of trace level radionuclides from aqueous solutions Science China Chemistry. 2015. Vol. 58. N 11. P. 1766. doi 10.1007 / s11426-015-5435-5

4. Jeon I.Y., Ju M.J., Xu J., Choi H.J., Seo J.M., Kim M.J., Liu H.K. Edge-Fluorinated Graphene Nanoplatelets as High-Performance Electrodes for Dye-Sensitized Solar Cells and Lithium-Ion Batteries Adv. Funct. Mater. 2015. Vol. 25. N 8. P. 1170. doi 10.1002 / adfm.201403836

5. KS Novoselov, AK Geim, SV Morozov, D Jiang, Y Zhang, SV Dubonos, IV Grigorieva and AA Firsov Electric field effect in atomically thin carbon films, Science, 2004, 306 (5696), pp. 666-669, doi: 10.1126/science.1102896

6. Li Xuesong; Cai Weiwei; An Jinho; Kim Seyoung; Nah Junghyo; Yang Dongxing; Piner Richard; Velamakanni Aruna; Jung Inhwa; Tutuc Emanuel; Banerjee Sanjay K .; Colombo Luigi; Ruoff Rodney S. Large-Area Synthesis of High-Quality and Uniform Graphene Films on Copper Foils, Science, 2009, Vol. 324, Num. 5932, pp. 1312-1314, DOI:10.1126/science.1171245

7. Emtsev Konstantin V .; Bostwick Aaron; Horn Karsten; Jobst Johannes; Kellogg Gary L .; Ley Lothar; McChesney Jessica L ;; Ohta Taisuke; Reshanov Sergey A .; Roehrl Jonas; Rotenberg Eli; Schmid Andreas K .; Waldmann Daniel; Weber Heiko B .; Seyller Thomas, Towards wafer-size graphene layers by atmospheric pressure graphitization of silicon carbide, Nature Materials, 2009, Vol. 8, Num. 3, pp. 203-207, DOI https://doi.org/10.1038/nmat2382

8. Luo Jiayan; Cote Laura J .; Tung Vincent C .; Tan Alvin T.L .; Goins Philip E .; Wu Jinsong; Huang Jiaxing Graphene Oxide Nanocolloids, Journal of the American Chemical Society, 2009, Vol. 132, Num. 50, pp. 17667-17669, https://doi.org/10.1021/ja1078943

9. Paredes Paredes J.I .; Villar-Rodil S .; Martinez-Alonso A .; Tascon J.M.D. Graphene oxide dispersions in organic solvents, Langmuir, 2008, Vol. 24, Num. 19, pp. 10560-10564, DOI: 10.1021/la801744a

10. Brodie, B.C. XIII. On the atomic weight of graphite. // Philosophical Transactions of the Royal Society of London. 1859. V. 149. P.249-259.36, https://doi.org/10.1098/rstl.1859.0013

11. Hummers, W.S., Offeman, R.E. Preparation of Graphitic Oxide. Journal of the American Chemical Society. 1958. V. 80. N.6. P.1339.37, http://dx.doi.org/10.1021/ja01539a017

12. Ma, J., Ping, D., Dong, X. Recent Developments of Graphene Oxide-Based Membranes: A Review. Membranes. 2017. V.7. N.3. article number 52.38, https://doi.org/10.3390/membranes7030052

13. Ibrahim, A., Lin, Y.S. Gas permeation and separation properties of large-sheet stacked graphene oxide membranes. Journal of Membrane Science. 2018. V.550. P.238-245, https://doi.org/10.1021/jp804586q 
14. Sungjin Parka, Jinho An, Jeffrey R. Potts, Aruna Velamakanni, Shanthi Murali, Rodney S. Ruoff Hydrazine-reduction of graphite- and graphene oxide, Carbon, 2011, Vol. 49, pp. 3019-3023, doi:10.1016/j.carbon.2011.02.071

15. Dresselhaus, MS; Dresselhaus, G, Intercalation compounds of graphite, Advances in Physics, 2002, Volume 51, Issue 1, Pages 1-186, DOI: 10.1080 / 00018730110113644 ;

16. Yuqi Li, Yaxiang Lu, Philipp Adelhelm, Maria-Magdalena Titirici and Yong-Sheng Hu, Intercalation chemistry of graphite: alkali metal ions and beyond, Chem. Soc. Rev., 2019, 48, 4655-4687 doi: 10.1039 / c9cs00162j

17. Tang, Libin; Li, Xueming; Ji, Rongbin, Kar Seng Teng, Guoan Tai, Jing Ye, Changsong Wei and Shu Ping Lau, Bottom-up synthesis of large-scale graphene oxide nanosheets, Journal of Materials Chemistry, 2012, Volume 22, Issue 12, Pages 56765683, DOI: 10.1039/c2jm15944a

18. Marwa Adel and Azza El-Maghraby, Ossama El-Shazly and El-Wahidy F. El-Wahidy, Marwa AA Mohamed, Synthesis of few-layer graphene-like nanosheets from glucose: New facile approach for graphene-like nanosheets large-scale production, J. Mater. Res., 2016, Vol. 31, No. 4, pp. 455-467, DOI: 10.1557/jmr.2016.25

19. Sandeep Pandey, Manoj Karakoti, Sunil Dhali, Neha Karki, Boddepalli SanthiBhushan, Chetna Tewari, Sravendra Rana, Anurag Srivastava, Anand B. Melkani, Nanda Gopal Sahoo, Bulk synthesis of graphene nanosheets from plastic waste method: waste management for better tomorrow, Waste Management, 2019, Vol. 88, pp. 48-55, https://doi.org/10.1016/j.wasman.2019.03.023

20. Qingfeng Yang, Ming Zhou, Mingyang Yang, Zhixun Zhang, Jianwen Yu, Yibo Zhang, Wenjun Cheng and Xuyin Li, HighYield Production of Few-Layer Graphene via New-fashioned Strategy Combining Resonance Ball Milling and Hydrothermal Exfoliation, 2020 , Vol. 10, pp. 667, http://dx.doi.org/10.3390/nano10040667 and references therein

21. Taniya Purkait, Guneet Singh, Mandeep Singh, Dinesh Kumar \& Ramendra Sundar Dey, Large area few-layer graphene with scalable preparation from waste biomass for high-performance supercapacitor, Scientific Reports, 2017 , Vol. 7, pp. 15239, doi: 10.1038 / s41598-017-15463-w

22. A.B. Volkova, E.V. Rezchikova, V.A. Shakhnov. Eng. J .: Sci. Innovation, 2013, 18 (6), 11; Reina, A., Jia, X., Ho, J., Nezich, D., Son, H., Bulovic, V., Kong, J. Large Area, Few-Layer Graphene Films on Arbitrary Substrates by Chemical Vapor Deposition. Nano Letters, 2009, 9 (1), 30-35. doi: 10.1021 / nl801827v

23. Merzhanov, AG. The chemistry of self-propagating high-temperature synthesis, Journal of Materials Chemistry, 2004, Vol. 14, N. 12, pp. 1779-1786, doi: 10.1039 / b401358c

24. Azatyan, V. V. Non-Isothermal Regimes and Chemical Control of Branching-Chain Processes. Russ. Chem. Rev. 1999, 68, 1021-1039. DOI: 10.1070/RC1999v068n12ABEH000520

25. A.E. Sytschev and A. G. Merzhanov. Self-propagating high-temperature synthesis of nanomaterials, Russ. Chem. Rev., 2004, 73 (2), 147, http://dx.doi.org/10.1070/RC2004v073n02ABEH000837

26. Alexander S. Rogachev, Alexander S. Mukasyan, Combustion for Material Synthesis, CRC Press, 2015, Taylor \& Francis Group, 6000 Broken Sound Parkway NW, Suite 300, Boca Raton, FL 33487-2742, 2015, 424 Pages, https://doi.org/10.1201/b17842

27. S. M. Krutov, A. P. Voznyakovskii, I. V. Gribkov, I. V. Shugalei. Lignin Wastes: Past, Present, and Future // Russian Journal of General Chemistry, 2014. Vol 84. No. IS. pp. 2632-2642, https://doi.org/10.1134/S1070363214130222

28. Mohan Dinesh, Pittman Charles U., Jr .; Steele, Philip H., Pyrolysis of wood / biomass for bio-oil: A critical review, Energy \& Fuels, 2006, Vol. 20, Num. 3, pp. 848-889, DOI: 10.1021 / ef0502397

29. Ferrari A.C. Meyer J.C. Scardaci V. Casiraghi C., Lazzeri M., Mauri F., Piscanec S., Jiang D., Novoselov K.S., Roth S., Raman spectrum of graphene and graphene layers, Physical Review Letters, 2006, Vol. 97, Num. 18, p. 187401, DOI: 10.1103 / PhysRevLett. 97.187401

30. Cunguang Lou, Shuo Wang, Tie Liang, Chenyao Pang, Lei Huang, Mingtao Run and Xiuling Liu, A Graphene-Based Flexible Pressure Sensor with Applications to Plantar Pressure Measurement and Gait Analysis, Materials, 2017, 10 (9), 1068 ; https://doi.org/10.3390/ma10091068

31. Stobinski L., Lesiak B., Malolepszy A., Mazurkiewicz M., Mierzwa B., Zemek J., Jiricek P., Bieloshapk I. // J. Electron Spectrosc. Relat. Phenom. 2014. Vol. 195. P. 145. doi 10.1016 / j.elspec.2014.07.003

32. Johra F.T., Lee J.W., Jung W.G. / Facile and safe graphene preparation on solution based platform / J. Ind. Eng. Chem. 2014. Vol. 20. P. 2883. doi 10.1016 / j.jiec.2013.11.022

33. Seung Hun Huh,X-ray diffraction of multi-layer graphenes: Instant measurement and determination of the number of layers, Carbon, 2014, Vol. 78, p. 617-621. 10.1016/j.carbon.2014.07.034

34. Patterson A.L., The Scherrer formula for X-Ray particle size determination, Phys. Rev., 1939, Vol. 56, No. 10, p. 978-982, https://doi.org/10.1103/PhysRev.56.978

35. J.G. Um, Y.S. Jun, H. Alhumade, H. Krithivasan, G. Lui, A. Yu, Investigation of the size effect of graphene nano-platelets (GNPs) on the anti-corrosion performance of polyurethane/GNP composites, RSC advances, 2018, Vol. 8, no.31, p. 1709117100, https://doi.org/10.1039/C8RA02087F

36. A.R. Ravindran, C. Feng, S. Huang, Y. Wang, Z. Zhao, J. Yang, Effects of graphene nanoplatelet size and surface area on the AC electrical conductivity and dielectric constant of epoxy nanocomposites, Polymers, 2018, Vol. 10, No. 5, p. 477, https://doi.org/10.3390/polym10050477

37. Claudia Backes, Keith R. Paton, Damien Hanlon, Shengjun Yuan, Mikhail I. Katsnelson, James Houston, Ronan J. Smith, David McCloskey, John F. Donegan, Jonathan N. Coleman, Spectroscopic metrics allow in situ measurement of mean size and thickness of liquid- exfoliated few-layer graphene nanosheets. Nanoscale, 2016, vol. 8, pp. 4311-4323, https://doi.org/10.1039/C5NR08047A 
38. Li Zhang, Wenfeng Zhu, Ying Huang and Shuhua Qi, Synergetic Effects of Silver Nanowires and Graphene Oxide on Thermal Conductivity of Epoxy Composites, nanomaterials, 2019, vol. 9, p. 1264, https://doi.org/10.3390/nano9091264 